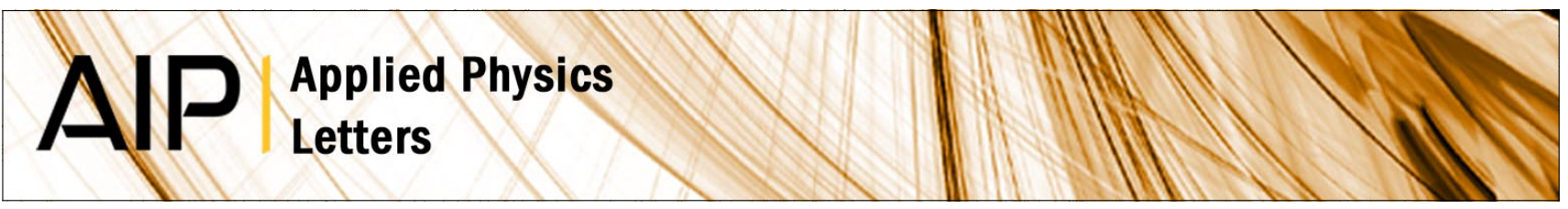

\title{
Evidence for hydrogen generation in laser- or spark-induced cavitation bubbles
}

Takehiko Sato, Marc Tinguely, Masanobu Oizumi, and Mohamed Farhat

Citation: Appl. Phys. Lett. 102, 074105 (2013); doi: 10.1063/1.4793193

View online: http://dx.doi.org/10.1063/1.4793193

View Table of Contents: http://apl.aip.org/resource/1/APPLAB/v102/i7

Published by the American Institute of Physics.

\section{Related Articles}

Nonlinear absorption and harmonic generation of laser in a gas with anharmonic clusters

Phys. Plasmas 20, 023302 (2013)

Analysis of spatially resolved Z-pinch spectra to investigate the nature of "bright spots"

Phys. Plasmas 20, 022707 (2013)

Two-dimensional space-resolved emission spectroscopy of laser ablation plasma in water

J. Appl. Phys. 113, 053302 (2013)

Direct evidence of mismatching effect on $\mathrm{H}$ emission in laser-induced atmospheric helium gas plasma J. Appl. Phys. 113, 053301 (2013)

"Water window" sources: Selection based on the interplay of spectral properties and multilayer reflection bandwidth

Appl. Phys. Lett. 102, 041117 (2013)

\section{Additional information on Appl. Phys. Lett.}

Journal Homepage: http://apl.aip.org/

Journal Information: http://apl.aip.org/about/about_the_journal

Top downloads: http://apl.aip.org/features/most_downloaded

Information for Authors: http://apl.aip.org/authors

\section{ADVERTISEMENT}

\section{AIP Applied Physics Letters}

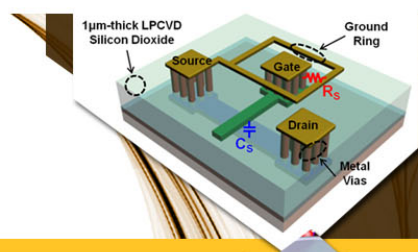

\section{SURFACES AND} INTERFACES

Focusing on physical, chemical, biological structural, optical, magnetic and electrical properties of surfaces and interfaces, and more...

\section{EXPLORE WHAT'S NEW IN APL}

SUBMIT YOUR PAPER NOW!
ENERCY CONVERSION AND STORACE 


\title{
Evidence for hydrogen generation in laser- or spark-induced cavitation bubbles
}

\author{
Takehiko Sato, ${ }^{1, a)}$ Marc Tinguely, ${ }^{2}$ Masanobu Oizumi, ${ }^{3, b)}$ and Mohamed Farhat ${ }^{2}$ \\ ${ }_{1}^{1}$ Institute of Fluid Science, Tohoku University, 2-1-1 Katahira, Aoba-ku, Sendai 980-8577, Japan \\ ${ }^{2}$ Laboratory for Hydraulic Machines, Swiss Federal Institute of Technology Lausanne (EPFL), \\ Avenue de Cour 33 bis, 1007 Lausanne, Switzerland \\ ${ }^{3}$ NS PLANT DESIGNING Corp., 46-59 Nakahara, Tobata-ku, Kitakyushu 804-0002, Japan
}

(Received 23 October 2012; accepted 7 February 2013; published online 21 February 2013)

\begin{abstract}
The growing use of focused lasers or electric sparks to generate cavitation bubbles raises concerns about the possible alteration of gas content during the initiation process and its effect on bubble dynamics. We provide experimental evidence that hydrogen molecules are produced for such plasma-induced bubbles. We performed spectral analysis of the light emitted by the plasma and monitored the dissolved hydrogen concentration in water. The mass of dissolved hydrogen was found proportional to the potential energy of the rebound bubble for both laser and spark methods. Nevertheless, hydrogen concentration was found 2.7 times larger with the spark. (C) 2013 American Institute of Physics. [http://dx.doi.org/10.1063/1.4793193]
\end{abstract}

Cavitation bubbles are used in various areas, including medical treatment, ${ }^{1,2}$ environmental treatment, ${ }^{3}$ and surface cleaning, ${ }^{4}$ where the phenomena occurring at the collapse of the bubble ${ }^{2,5,6}$ are taken advantage of. To understand these phenomena, namely, shock waves, microjets, chemical reactions, luminescence, and nano/microbubbles, most experimental studies on the dynamics of cavitation bubbles in still water ${ }^{6-8}$ use point-like hot plasma which is used to initiate thermal growth of a quasi-spherical vapor bubble in a controlled and repetitive way. Such plasma is obtained either by focusing a pulsed laser ${ }^{9}$ or by producing an electric spark between two immersed electrodes. ${ }^{10,11}$ As it expands, the bubble cools down and reaches a maximum radius, which is limited by the initial energy in the plasma and the pressure within the liquid. The bubble then undergoes a collapse phase in good agreement with the Rayleigh model. ${ }^{12}$ At the final stage of the collapse, both the pressure and the temperature increase significantly within the bubble with the possibility of complex chemical reactions, which may influence the water condensation process at the bubble interface. ${ }^{13}$ The water vapor could be trapped within the cavity and become compressed as well. ${ }^{13-15}$ It is widely believed that under these extreme conditions, the amount of gas present in the cavity is a crucial parameter that governs all the relevant phenomena associated with the bubble collapse. The size of the rebound and the energy of the shock wave are influenced by the amount of non-condensable gas in the bubble ${ }^{16}$ and the emission intensity of sonoluminescence depends on the type of trapped gases, such as inert gases and air, in the collapsing bubble. ${ }^{17,18}$ The gas trapped in the bubble is closely related to the gas dissolved in water, because diffusion occurs during the oscillation process. Therefore, the monitoring of dissolved gas in water gives an indication of the amount and type of gas within the bubble.

\footnotetext{
${ }^{\text {a)} E-m a i l: ~ s a t o @ i f s . t o h o k u . a c . j p . ~}$

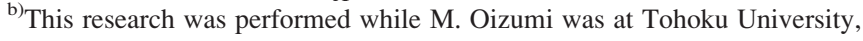
Sendai 980-8577, Japan.
}

It is widely accepted that laser- or spark-induced bubbles behave like hydrodynamic cavitation bubbles, which can form in flowing water. It is commonly argued that when the maximum radius is reached, thermodynamic equilibrium is recovered and the bubble dynamics are no longer influenced by the initial hot plasma. Owing to this assumption, tremendous progress has been made in understanding single cavitation bubble dynamics. Nevertheless, this assumption may be questionable because of the possible occurrence of chemical reactions during plasma generation with subsequent changes in the gas content within the bubble. Indeed, the plasma generated by the laser or the spark can reach a temperature that is high enough for hydrogen to form through the chemical process of water dissociation. . $^{3,19,20}$

In this study, measurement of the dissolved hydrogen concentration and spectral analysis of the light emitted by the plasma were performed during the successive collapses and rebounds of both laser- and spark-induced bubbles to detect and quantify the presence of hydrogen and to understand the key factors of hydrogen generation.

Figure 1 shows the experimental setup. It consisted of a vessel, a laser source, an optical arrangement for laser beam focusing, electrodes with a power supply, a high-speed camera, and a delay line for synchronization purposes. Laser-

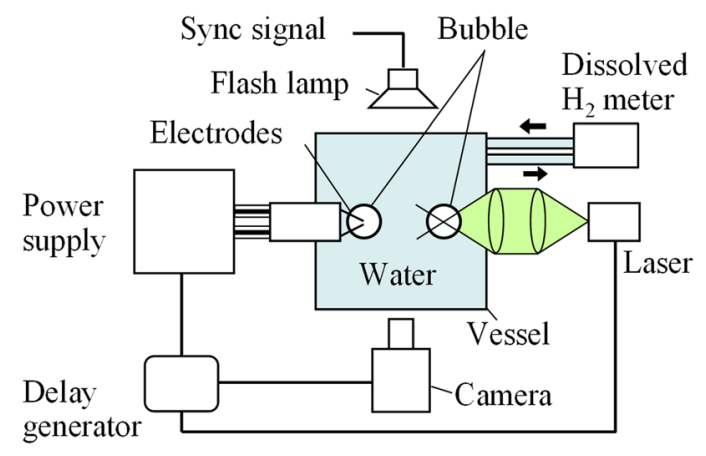

FIG. 1. Schematic of the experimental setup for observation of bubble dynamics and measurement of dissolved hydrogen. 
induced bubbles were generated by a Q-switch Nd:YAG laser providing a 5-ns pulse at a wavelength of $532 \mathrm{~nm}^{21}$ The energy per pulse, $E_{\text {laser }}$, was varied between 3.2 and 10.6 mJ. For spark-induced bubbles, platinum electrodes with a diameter of $0.3 \mathrm{~mm}$ and a gap of $0.075 \mathrm{~mm}$ were used. The spark energy per pulse, $E_{\text {spark }}$, was $0.32,0.76$, or $1.2 \mathrm{~J}^{22}$ The vessel was filled with $250 \mathrm{ml}$ of ultrapure water (Millipore, Simplicity UV). The bubbles were observed using a highspeed camera (Photron, Fastcam SA1.1) at 100000 fps and 1 $\mu$ s of exposure time, with a resolution of $320 \times 128$ pixels. The emission lines during plasma generation were analyzed with an optical multichannel analyzer (Hamamatsu Photonics, PMA-12). A notch filter with a center wavelength of $532 \mathrm{~nm}$ and a full-width at half-maximum of $10 \mathrm{~nm}$ was used to reduce the intensity of laser scattering. The notch filter also cuts off the emissions with wavelengths shorter than $320 \mathrm{~nm}$. The concentration of dissolved $\mathrm{H}_{2}$ was measured with a polarographic-type sensor with a resolution of $0.01 \mu \mathrm{g} \cdot 1^{-1}$ (Bionics-kiki, BIH-50D) by circulating the water at a flow rate of $0.91 \cdot \mathrm{min}^{-1}$. The water was changed for each new test to avoid residual dissolved $\mathrm{H}_{2}$. This device was equipped with a diaphragm to prevent microbubbles and thus ensure the reliability of the measurements. For each experimental condition, the tests were repeated at least 3 times to allow the results to be averaged. These experiments were performed at atmospheric pressure and room temperature $\left(\sim 20^{\circ} \mathrm{C}\right)$.

The Rayleigh Eq. (1) ${ }^{6,12}$ was used to compare the experimental results with those of a theoretical model

$$
R \ddot{R}+\frac{3}{2} \dot{R}^{2}=\frac{P_{v}-P_{\infty}}{\rho} .
$$

Here $R$ is the bubble radius, which depends on time, $\rho$ is the water density, and $P_{v}$ and $P_{\infty}$ are, respectively, vapor pressure and pressure in the surrounding water. Rayleigh time $T_{\text {Rayl }}$ in Eq. (2) is the collapse time of the bubble, i.e., the time between bubble maximum diameter and the first collapse of the bubble.

$$
T_{\text {Rayl }}=k R_{\max } \sqrt{\rho /\left(P_{\infty}-P_{v}\right)} .
$$

Here $k \approx 0.915$ is a constant, and $R_{\max }$ is the maximum radius of the bubble. The radii of the bubbles were estimated from the movies using cross-sectional area $A_{\mathrm{b}}\left(R=\left(A_{\mathrm{b}} / \pi\right)^{1 / 2}\right)$.

Figure 2(a) presents snapshots of bubbles for the laser and spark cases at different key stages: (1) initiation, (2) maximum radius, (3) main collapse, and (4) end of successive collapses and rebounds. The evolution of the normalized radius of the bubbles is shown in Fig. 2(b). Bubble radius $R$ and time $t$ were normalized, respectively, by the maximum bubble radius $R_{\max }$ and the Rayleigh time $T_{\text {Rayl. }}$. The solid line shows the evolution of the normalized radius of the bubble predicted by the Rayleigh equation. In the case of the laser, after a few weak rebounds and collapses, the volume of the residual gas quickly reaches a steady value $R^{*}=R / R_{\max }=0.08$ within a time frame of $t^{*}=t / T_{\text {Rayl }}=3$. In the case of the spark, the bubble oscillates longer, with larger amplitude. It takes almost $t^{*}=6$ for the bubble to reach a steady radius of $R^{*}=0.21$. These results show that despite similar collapse processes that
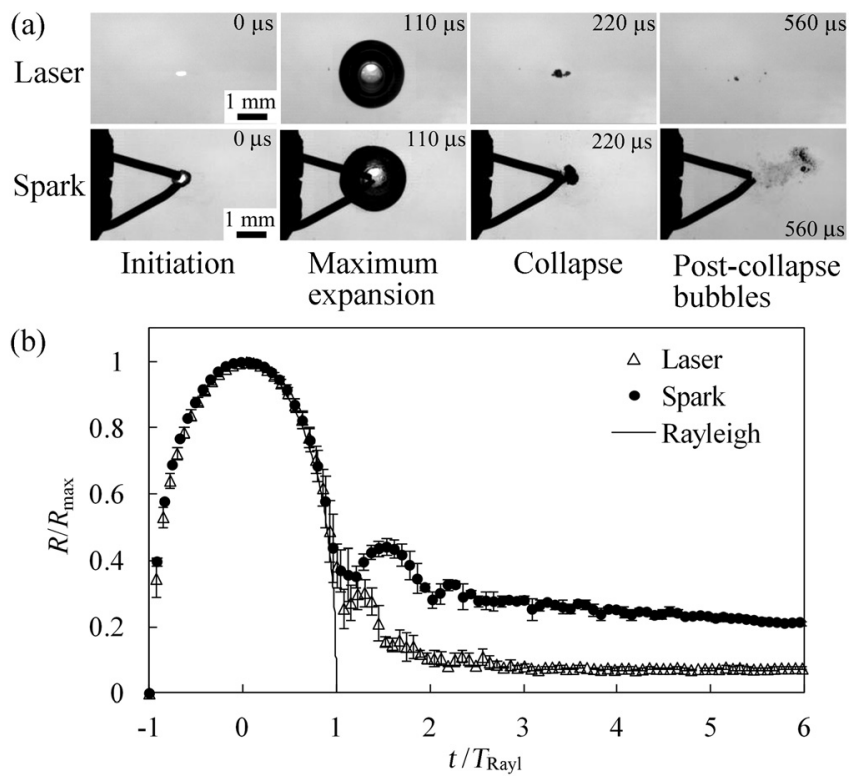

FIG. 2. Visualization of bubble behaviors for the laser and spark cases at the initiation, maximum expansion, collapse, and post-collapse of bubbles (a); the time evolution of the reduced radius of bubbles for the both cases, and comparison with Rayleigh theory (b). The open triangles and closed circles denote the laser case and the spark case, respectively. The error bars denote standard error.

agree with the Rayleigh theory, differences appear both in the number of post-collapse bubbles and in the dynamics of the rebound when the bubbles are generated either by a laser or by a spark.

Figure 3 shows the emission spectra at the generation of the bubbles for the laser case (a) and the spark case (b) revealing the generation of hydrogen atoms. By black body radiation curve fitting, shown by the dashed lines in Figs. 3(a) and 3(b), temperatures of around $11000 \mathrm{~K}$ for the laser-induced bubbles and around $14000 \mathrm{~K}$ for the sparkinduced bubbles were obtained. Those estimated temperatures agree with values reported in the literature. ${ }^{23}$ With such high temperatures, $\mathrm{H}$ and $\mathrm{OH}$ were likely generated by thermal dissociation of water vapor. ${ }^{20,24,25}$ The relationships between the mass of the dissolved $\mathrm{H}_{2}$ per pulse and the radiation intensities of $\mathrm{H}_{\beta}$ and $\mathrm{H}_{\alpha}$ lines observed in the laser case and the spark case are also shown in Figs. 3(c) and 3(d), respectively. Here, the intensity is the difference between the measured intensity and the intensity obtained from the fitting black body curve. Since the mass of generated hydrogen gas increased with the increase of the radiation intensities of $\mathrm{H}_{\beta}$ and $\mathrm{H}_{\alpha}$ in both cases, these results also provide evidence that the generated hydrogen atoms at the plasma spot change into hydrogen molecules due to recombination.

The generation of hydrogen gas was also verified by measuring the concentration of dissolved $\mathrm{H}_{2}$. Figure 4(a) shows the mass of dissolved $\mathrm{H}_{2}$ divided by the potential energy of the bubble and the number of pulses versus the potential energy of a bubble for different output laser energies. Figure 4(b) shows the mass versus the potential bubble energy for different spark energies. The potential energy of a bubble at its maximum radius, $E_{\mathrm{b}}$, were derived by $E_{b}=4 \pi R_{\max }^{3}\left(p_{\infty}-p_{v}\right) / 3{ }^{26}$ In the cases of both laserinduced bubbles and spark-induced bubbles, the mass of 

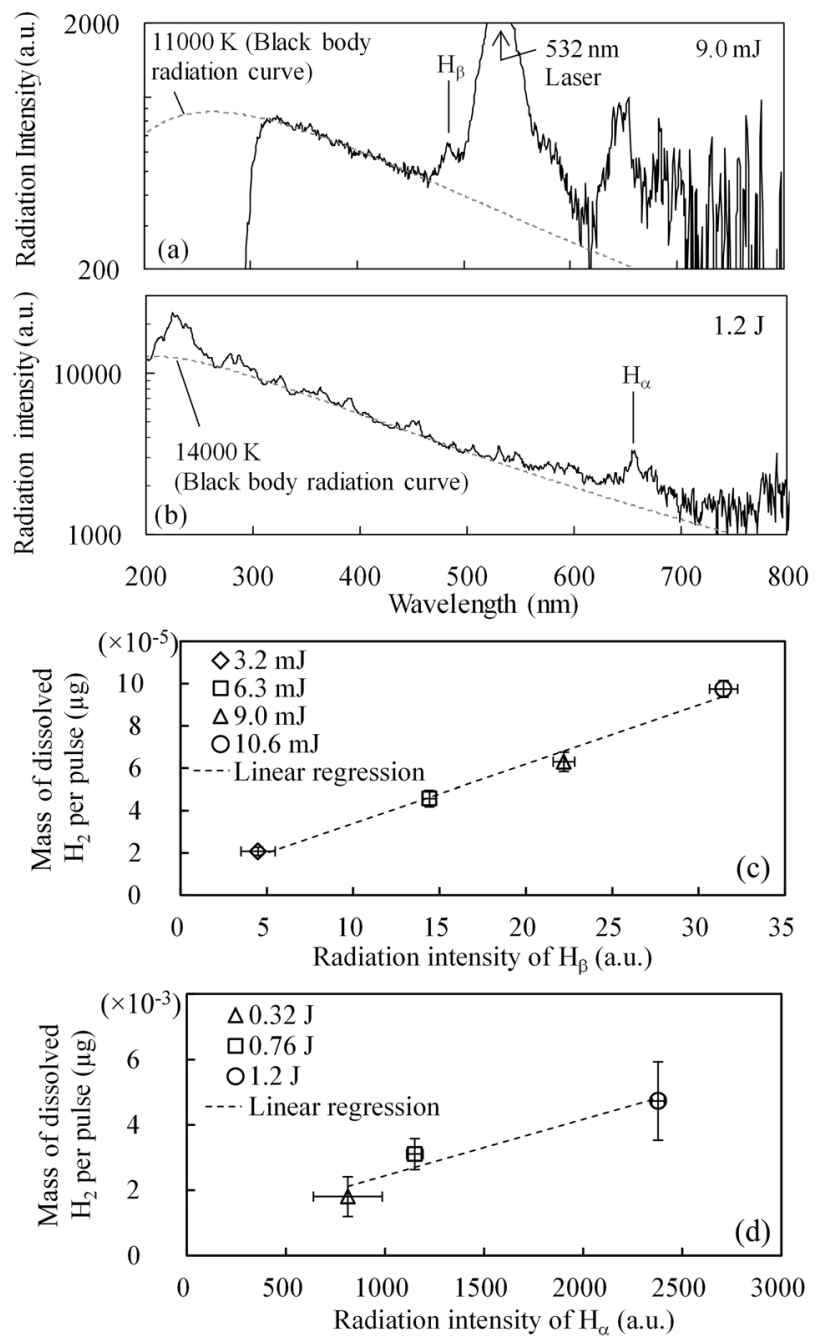

FIG. 3. Spectral analysis at the initiation of the bubbles and the black body fittings for the laser case at $9.0 \mathrm{~mJ}$ (a) and the spark case at $1.2 \mathrm{~J}$ (b). The mass of dissolved $\mathrm{H}_{2}$ per pulse increases proportional to the radiation intensities of $\mathrm{H}_{\beta}$ and $\mathrm{H}_{\alpha}$ lines observed in the laser case (c) and the spark case (d). The error bars denote standard error.

dissolved $\mathrm{H}_{2}$ was almost constant against the potential energy of the bubble. This hydrogen gas was possibly generated by chemical reactions such as $\mathrm{H}_{2} \mathrm{O}_{2}+\mathrm{H} \rightarrow \mathrm{HO}_{2}+\mathrm{H}_{2}$, $\mathrm{HO}_{2}+\mathrm{H} \rightarrow \mathrm{H}_{2}+\mathrm{O}_{2}$ and $\mathrm{H}+\mathrm{H}+\mathrm{H}_{2} \mathrm{O} \rightarrow \mathrm{H}_{2}+\mathrm{H}_{2} \mathrm{O},{ }^{25}$ which resulted from the generation of hydrogen atoms and chemical species by the thermal dissociation of water vapor due to the high temperature at the initiation. Hydrogen molecules can also be formed by both $\mathrm{H}$ radical recombination and hydroxyl radical attack through $\mathrm{H}+\mathrm{OH} \rightarrow \mathrm{O}+\mathrm{H}_{2} \cdot{ }^{27}$

The mean mass of dissolved hydrogen, $M_{\mathrm{H} 2}$, divided by the potential energy of the bubble was 2.7 times larger for the spark case $\left(M_{\mathrm{H} 2} / E_{\mathrm{b}}=0.17 \mu \mathrm{g} \cdot \mathrm{J}^{-1}\right)$ than for the laser case $\left(M_{\mathrm{H} 2} / E_{\mathrm{b}}=0.063 \mu \mathrm{g} \cdot \mathrm{J}^{-1}\right)$. This difference can be explained by the involvement of additional physical phenomena when the plasma was initiated by a spark. In the case of the spark, in addition to thermal dissociation, electrolysis $\left(2 \mathrm{H}_{2} \mathrm{O}(l)\right.$ $\left.\rightarrow 2 \mathrm{H}_{2}(g)+\mathrm{O}_{2}(g)\right)^{28}$ and electron impact dissociation of water molecules $\left(e+\mathrm{H}_{2} \mathrm{O} \rightarrow e+\mathrm{H}+\mathrm{OH}\right)^{29}$ occurred, which led to the formation of a larger mass of $\mathrm{H}_{2}$. Note that these phenomena could also explain the difference in the bubble generation energy efficiencies because they represent the sources of energy dissipation specific to the spark case. In
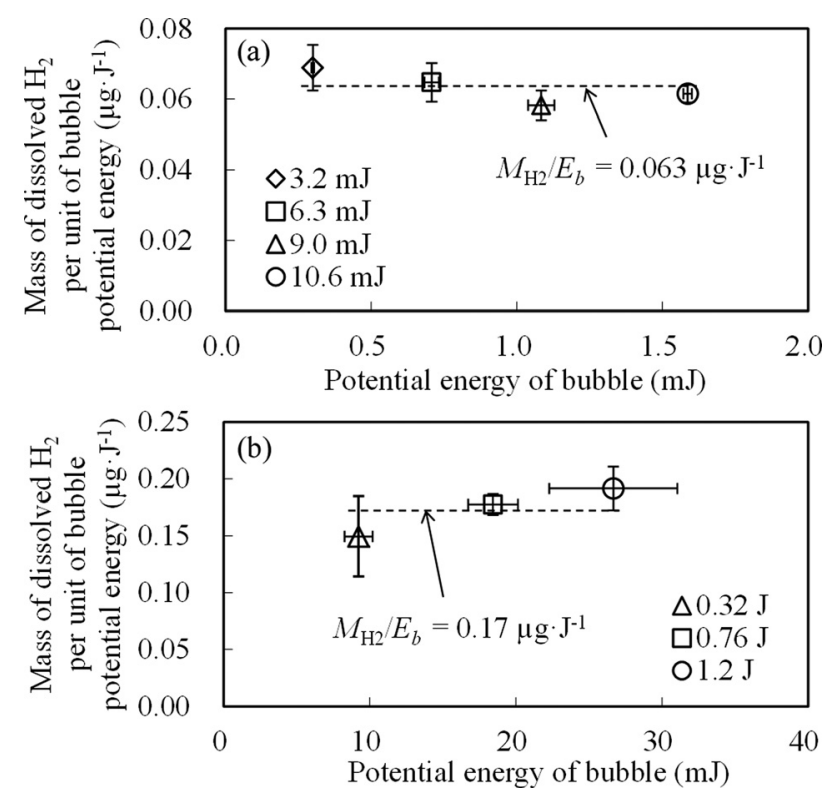

FIG. 4. Mass of dissolved $\mathrm{H}_{2}$ per unit of bubble potential energy vs. potential energy of the bubble for different laser (a) and spark (b) energies. In the laser case, each point was the average of 5 samples, each of the 5 samples being the average of 3 samples for different numbers of pulses such as 100 , $200,300,400$, and 500. In the spark case, each point was the average of 10 or 11 samples. The error bars denote standard error.

the case of the laser, $12 \%$ of the input energy is converted into the bubble $\left(E_{\mathrm{b}} / E_{\text {laser }}\right)$, whereas for the spark, only $2.5 \%$ of the input energy is converted into the bubble $\left(E_{\mathrm{b}} / E_{\text {spark }}\right)$.

Figure 5 shows the potential energy of a rebound bubble versus the mass of dissolved $\mathrm{H}_{2}$ per pulse in the cases of the laser and the spark. The potential energy of the rebound bubble increased with the increase in generated hydrogen gas. The linear regression going through the origin shows a good relationship for both the laser and the spark cases, especially when the mass of $\mathrm{H}_{2}$ is greater than $5 \times 10^{-5} \mu \mathrm{g}$. This result means that there is a universal relationship between the potential energy of rebound bubbles and the mass of dissolved $\mathrm{H}_{2}$ and provides further evidence of the effect of hydrogen gas on cavitation bubble dynamics.

In conclusion, the mass of hydrogen increases proportionally with the potential energy of the bubble, but the factor of proportionality depends on how the bubble is generated,

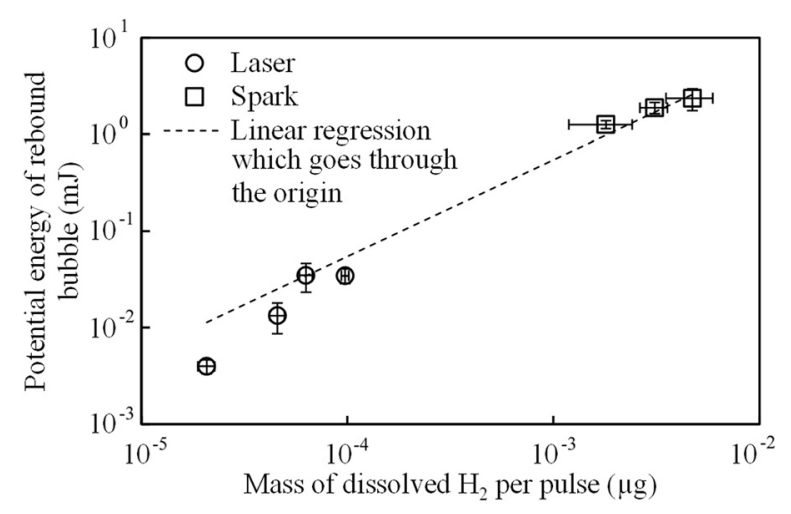

FIG. 5. Potential energy of rebound bubble vs. the mass of dissolved $\mathrm{H}_{2}$ per pulse in the case of the laser and the spark. The linear regression goes through the origin, i.e., $M_{\mathrm{H} 2}=0 \mu \mathrm{g}$ and $E_{\mathrm{b} \_ \text {rebound }}=0 \mathrm{~mJ}$. The error bars denote standard error. 
i.e., laser or spark. By contrast, the potential energy of the rebound bubble is proportional to the mass of hydrogen with a universal constant of proportionality that does not depend on whether a laser or spark was used to generate the bubble. The mass of hydrogen increased with the intensities of the hydrogen emission lines in both the laser and spark cases; it is believed that hydrogen molecules are generated at the initiation of the bubble by the occurrence of thermal dissociation. It was also found that the mass of hydrogen for the spark-induced bubbles was 2.7 times greater than that for the laser-induced bubbles. This difference can be explained by the occurrence of electron impact dissociation and electrolysis in the spark case. The potential energy of the rebound bubble was proportional to the mass of dissolved hydrogen in both the laser and spark cases. These results are the evidence of the presence of hydrogen molecules in the bubble and contribute to the widely discussed question regarding the difference between "hydrodynamic" and laser- or spark-induced cavitation bubbles.

This study was partly supported by JSPS Core-to-Core Project (No. 20001), by Toyo Advanced Technologies, Co., by the Collaborative Research Project of IFS, Tohoku University, by the JSPS Grant-in-aid for Scientific Research, and by the Swiss National Science Foundation (Grant No. 200020116641). We wish to thank François Avellan, Makoto Ohta, Tatsuyuki Nakatani, and Tomoki Nakajma for their help.

${ }^{1}$ B. J. O'Daly, E. Morris, G. P. Gavinc, J. M. O'Byrne, and G. B. McGuinness, J. Mater. Process. Technol. 200, 38 (2008).

${ }^{2}$ D. A. Fletcher and D. V. Palanker, Appl. Phys. Lett. 78, 1933 (2001).

${ }^{3}$ O. L. Li, N. Takeuchi, Z. He, Y. Guo, K. Yasuoka, J. S. Chang, and N. Saito, Plasma Chem. Plasma Process. 32, 343 (2012).
${ }^{4}$ C.-D. Ohl, M. Arora, R. Dijkink, V. Janve, and D. Lohse, Appl. Phys. Lett. 89, 074102 (2006).

${ }^{5}$ B. Gerold, P. Glynne-Jones, C. McDougall, D. McGloin, S. Cochran, A. Melzer, and P. Prentice, Appl. Phys. Lett. 100, 024104 (2012).

${ }^{6}$ Fundamentals of Cavitation, edited by J. P. Franc and J. M. Michel (Kluwer Academic, Dordrecht, 2004).

${ }^{7}$ M. S. Plesset and A. Prosperetti, Annu. Rev. Fluid Mech. 9, 145 (1977).

${ }^{8}$ J. R. Blake and D. C. Gibson, Annu. Rev. Fluid Mech. 19, 99 (1987).

${ }^{9}$ A. Philipp and W. Lauterborn, J. Fluid Mech. 361, 75 (1998).

${ }^{10}$ D. Obreschkow, P. Kobel, N. Dorsaz, A. Bosset, C. Nicollier, and M. Farhat, Phys. Rev. Lett. 97, 094502 (2006).

${ }^{11}$ S. Buogo and G. B. Cannelli, J. Acoust. Soc. Am. 111, 2594 (2002).

${ }^{12}$ L. Rayleigh, Philos. Mag. 34, 94 (1917).

${ }^{13}$ A. J. Colussi and M. R. Hoffmann, J. Phys. Chem. A 103, 11336 (1999).

${ }^{14}$ G. Hauke, D. Fuster, and C. Dopazo, Phys. Rev. E 75, 066310 (2007).

${ }^{15}$ I. Akhatov, O. Lindau, A. Topolnikov, R. Mettin, N. Vakhitova, and W. Lauterborn, Phys. Fluids 13, 2805 (2001).

${ }^{16}$ M. Tinguely, D. Obreschkow, P. kobel, N. Dorsaz, A. de Bosset, and M. Farhat, Phys. Rev. E 86, 046315 (2012).

${ }^{17}$ D. J. Flannigan and K. S. Suslick, Nature (London) 434, 52 (2005).

${ }^{18}$ M. Farhat, A. Chakravarty, and J. E. Field, Proc. R. Soc. A 467, 591 (2011).

${ }^{19}$ M. J. Kirkpatrick and B. R. Locke, Ind. Eng. Chem. Res. 44, 4243 (2005).

${ }^{20}$ G. Maatz, A. Heisterkamp, H. Lubatschowski, S. Barcikowski, C. Fallnich, H. Welling, and W. Ertmer, J. Opt. A: Pure Appl. Opt. 2, 59 (2000).

${ }^{21}$ E. Robert, J. Lettry, M. Farhat, P. A. Monkewitz, and F. Avellan, Phys. Fluids 19, 067106 (2007).

${ }^{22}$ F. Pereira, M. Farhat, and F. Avellan, in Proceedings of 1993 IUTAM Symposium on Bubble Dynamics and Interface Phenomena, Birmingham, U.K., 6-9 September 1993, pp. 227-240.

${ }^{23}$ O. Baghdassarian, H. C. Chu, B. Tabbert, and G. A. Williams, Phys. Rev. Lett. 86, 4934 (2001).

${ }^{24}$ H. Yui and T. Sawada, Phys. Rev. Lett. 85, 3512 (2000).

${ }^{25}$ S. Medodovic and B. R. Locke, J. Phys. D 40, 7734 (2007).

${ }^{26}$ B. Sun, M. Sato, and J. S. Clements, J. Electrost. 39, 189 (1997).

${ }^{27}$ K.-Y. Shih and B. R. Locke, IEEE Trans. Plasma Sci. 39, 883 (2011).

${ }^{28}$ M. A. Malik, A. Ghaffar, and S. A. Malik, Plasma Sources Sci. Technol. 10, 82 (2001).

${ }^{29}$ B. R. Locke and S. M. Thagard, Plasma Chem. Plasma Process. 32, 875 (2012). 